Article

\title{
The First Record of Monochamus saltuarius (Coleoptera; Cerambycidae) as Vector of Bursaphelenchus xylophilus and Its New Potential Hosts in China
}

\author{
Min Li ${ }^{1}{ }^{1}$, Huan Li ${ }^{1}$, Ruo-Cheng Sheng ${ }^{1}$, Hui Sun ${ }^{1}{ }^{(\mathbb{C}}$, Shou-Hui Sun ${ }^{2}$ and Feng-Mao Chen ${ }^{1, *}$ \\ 1 Co-Innovation Center for the Sustainable Forestry in Southern China, College of Forestry, Nanjing Forestry \\ University, Nanjing 210037, China; 1m950330@njfu.edu.cn (M.L.); lhuan@njfu.edu.cn (H.L.); \\ rcsheng@njfu.edu.cn (R.-C.S.); hui.sun@njfu.edu.cn (H.S.) \\ 2 College of Forestry, Shenyang Agricultural University, Shenyang 110866, China; sysshh@163.com \\ * Correspondence: cfengmao@njfu.edu.cn
}

Received: 20 August 2020; Accepted: 15 September 2020; Published: 16 September 2020

Simple Summary: During a survey of pine wilt disease in 2017, thousands of dead pitch pines (Pinus koraiensis) were discovered in Fengcheng, Liaoning Province, China. Samples were taken from several regions of the bole, and Bursaphelenchus xylophilus was collected from the dead pitch pine samples. Monochamus saltuarius is an indigenous insect in this area, but there is no experimental evidence that $M$. saltuarius can transport B. xylophilus in China. The experimental results showed that M. saltuarius was the new vector of pine wilt disease in China. In laboratory experiments, $M$. saltuarius fed and transmitted B. xylophilus not only on pines but also on other conifers. Taxus, Juniperus, Sabina and Podocarpus are new tree species upon which B. xylophilus can be fed and transmitted by M. saltuarius. Picea pungens, Picea asperata, Abies fabri, A. holophylla, Larix principis-rupprechtii, L. olgensis, Juniperus formosana, Sabina chinensis and Podocarpus macrophyllus are new species of coniferous trees known to be able to be infected by B. xylophilus in a laboratory setting. This study raises awareness to prevent the disease from expanding and provides a reference for the control of pine wilt disease.

\begin{abstract}
Pine wilt disease was first discovered in Dongtang town, Liaoning Province, China, in 2017. However, no record of Monochamus alteratus existed in Fengcheng, where M. saltuarius is an indigenous insect, and no experimental evidence has thus far indicated that $M$. saltuarius can transport the Bursaphelenchus xylophilus in China. In this study, we investigated whether M. saltuarius is a vector of B. xylophilus in China. On the sixth day after eclosion, beetles began to transmit nematodes into the twigs. The transmission period of nematodes is known to be able to last for 48 days after beetle emergence. In laboratory experiments, M. saltuarius fed and transmitted B. xylophilus not only on pines but also on other non-Pinus conifers. The non-Pinus conifers preferred by M. saltuarius for feeding are Picea pungens, Picea asperata, and Abies fabri. The experimental results show that M. saltuarius functions as a vector of $B$. xylophilus in northeast China.
\end{abstract}

Keywords: conifer tree; pinewood nematode; pine sawyer; host plants

\section{Introduction}

Pine wilt disease (PWD) is caused by the pinewood nematode (PWN) Bursaphelenchus xylophilus (Steiner and Buhrer, 1934) Nickle, 1970. With the development of economic globalization and increases in human migration, many nonnative organisms have been introduced into various ecological communities [1]. In many cases, when no natural enemy is present and the living environment is 
favorable, exotic organisms are likely to cause considerable harm to the ecosystem into which they enter. The PWN is native to north America [1], and this nematode has caused serious pine disease after its introduction to new regions, especially in East Asian countries such as Japan [2], China [3] and Korea [4]. In China, the first case of Pinus thunbergii Parl. showing PWD and withering was found in Zijin Mountain, Nanjing, Jiangsu Province, in 1982 [5]. Despite many measures being implemented during the past four decades, PWD has spread quickly to other provinces, and an optimal treatment has not yet been developed for this disease [6].

Insects related to PWD worldwide include 21 species of Cerambycidae, 1 species of Buprestidae and 2 species of Curculionidae, but the main vectors of PWD are beetles of the genus Monochamus (Cerambycidae) $[7,8]$. To date, 13 beetles of the genus Monochamus have been shown to carry PWNs. In north America, Monochamus carolinesis Olivier [9], M. scutellatus Say [10], M. titillator Fabricius [11], M. obtusus Casey [12], M. notatus Drury [10], M. marmorator Kirby and M. mutator LeConte [13] are vectors of PWN. In Asia, M. alternatus Hope [14], M. saltuarius Gebler [15] and M. nitens Bates can carry and transfer PWNs. Throughout Europe, M. galloprovincialis Olivier is the only species that currently functions as a vector of PWNs [16], while M. sutor Linné [17] and M. urussovi Fischer-Waldheim [18] are also potential vectors of PWN. In Europe and North Africa, M. galloprovincialis $[19,20]$ has been reported to be the local PWN vector.

During a survey of PWD in 2017, thousands of dead pitch pines (Pinus koraiensis Sieb. et Zucc. Fl.) were discovered in Fengcheng, Liaoning Province and attracted the attention of the National Forestry and Grassland Administration of China [21]. In a 2017 trap survey, we only found M. saltuarius, a pine tree beetle. A large number of such beetles had colonized weakened and dying pine trees in Dongtang town, which is the epidemic area in Fengcheng. Monochamus saltuarius belongs to the family Cerambycidae, and the species has been found to carry PWNs under their elytra [22]. This scenario has been shown to be widespread in Japan and South Korea, and the species has been reported to carry and transmit PWNs [23,24]. However, this beetle is an indigenous insect in this area [25], and to our knowledge, no reports have indicated that M. saltuarius transmits PWNs in China.

The PWN is generally believed to be transmitted through timber, mainly by beetles emerging from PWN-infected trees. In Fengcheng, the timber industry generates a large revenue and provides employment opportunities for thousands of people. Thus, this disease has caused serious social and economic impacts in this area. Pinus koraiensis is one of the most important pine species in this area, but unfortunately, this pine is also the preferred host of PWN [26]. Although many studies have been conducted in the past century, no effective strategy has been found to manage PWD. In addition, other genera, such as Abies, Cedrus, Chamaecyparis, Larix, Pseudotesuga and Picea, are also considered suitable PWN hosts [27]. Many types of trees exist on the outskirts of Dongtang town in addition to P. koraiensis [26], P. tabuliformis Carr [28] and P. armandii Franch [29], which have been shown to be infested by PWNs; the city also hosts Larix principis-rupprechtii Mayr, L. olgensis Henry, Taxus chinensis (Pilger) Rehd, Picea pungens, Picea asperata Mast, Juniperus formosana Hayata, Sabina chinensis (L.) Ant, Abies fabri (Mast.) Craib, A. holophylla Maxim and Podocarpus macrophyllus (Thunb.) D. Don, which account for large proportions and are the main conifer species.

The purpose of this work was to (I) assess whether M. saltuarius can carry PWNs; (II) confirm whether nematodes can be transmitted by feeding; and (III) test whether M. saltuarius can feed on conifer trees other than pine trees and whether these tree species can be infected by PWNs.

\section{Materials and Methods}

\subsection{Source and Preservation of Dead Trees}

During the period from September to December 2017, 1200 P. koraiensis trees (25-30 years old) that had been killed by PWNs were felled in the Dongtang town region of Fengcheng, Liaoning Province, China. Thirty trees were cut into logs (1.0-1.2 m- in length) and transported to the Forest Pest Control and Quarantine Bureau of Fengcheng City where they were placed outdoors. Thereafter, the timber 
was kept in a top-covered shed field cage $(200 \times 250 \times 200 \mathrm{~cm})$ with sidewalls composed of 0.2 -cm metal mesh. The logs were watered every 3 days until the end of the observation period, and daily observations were recorded from early May to the end of August 2018.

\subsection{Experimental Conifers and Food Sources}

The following mature trees were obtained from the Fengcheng Nursery (Fengcheng, Liaoning Province; $40^{\circ} 28^{\prime} 7^{\prime \prime} \mathrm{N}, 124^{\circ} 03^{\prime} 18^{\prime \prime} \mathrm{W}$ ) and kept in an open-air location: 5-year-old $P$. koraiensis seedlings; 18-year-old L. principis, L. olgensis and T. chinensis seedlings; and 20-year-old Picea pungens, Picea asperata, J. formosana, S. chinensis, A. fabri, A. holophylla and Podocarpus macrophyllus seedlings. All plant material was collected from this nursery, and twigs used for beetle food were recut to lengths of $15 \mathrm{~cm}$ in the laboratory.

\subsection{Detection of PWN Carried by M. saltuarius}

As the beetles emerged, they were captured in a cage from the Forest Pest Control and Quarantine Bureau and sexed, and the surrounding temperature and humidity were recorded daily. The number of dispersed juveniles carried by each newly emerged beetle was estimated by a non-destructive method, i.e., stereomicroscope examination of both the first abdominal and metathoracic spiracular openings to determine whether or not they contained PWN [30]. Sixteen beetles were selected and sectioned after examination and any remaining nematodes in the adults were extracted by the Baermann funnel technique, which is, according to Shuo [31], the most efficient method by which to extract nematodes from a vector body. Then, approximately $13 \mathrm{~mL}$ of liquid was collected at the bottom of the funnel, and the nematodes were collected. The next step was to shake the sample well and count the number of nematodes under an inverted light microscope (Carl Zeiss Imager M2, 37,081 Gattingen, Germany). Three replications were conducted for each isolate.

For further verification, DNA was extracted from individual nematodes. The internal transcribed spacer (ITS) primers F194 (5'-CGTAACAAGGTAGCTGTAG-3') and 5368r (5'-TCCTCCGCTAAATG ATATG-3') were used to identify the nematodes [32,33]. The following reagents were required for this identification: protease K, 10x PCR buffer, 10x ExTaq buffer, and dNTP mixture, which were purchased from Takara Biomedical Technology Co., Ltd. (Beijing, China); 2x EasyTaq PCR SuperMix, which was purchased from Genscript Biomedical Technology Co., Ltd. (Nanjing, China); and $\mathrm{MgCl}_{2}$ and primers, which were synthesized by Sangon Biomedical Technology Co., Ltd. (Shanghai, China).

Nematodes extracted from beetles were selected under a Leica DM500 microscope and washed twice with $\mathrm{dd}_{2} \mathrm{O}$. A total of 10 nematodes were randomly selected for molecular identification. The nematodes were placed in a 1.5-mL centrifuge tube, and each centrifuge tube had one nematode with $8 \mu \mathrm{L}$ of nematode lysis buffer and $2 \mu \mathrm{L}$ of protease $\mathrm{K}$. The samples were incubated at $65^{\circ} \mathrm{C}$ for $35 \mathrm{~min}$, heated to $95^{\circ} \mathrm{C}$ for $7 \mathrm{~min}$, and centrifuged for $10 \mathrm{~s}$ to extract DNA. The total volume of the polymerase chain reaction (PCR) was $25 \mu \mathrm{L}$, including $2.5 \mu \mathrm{L}$ of $10 \times$ PCR buffer, $2 \mu \mathrm{L}$ of $2.5 \mathrm{mmol} / \mathrm{L}$ $\mathrm{MgCl}_{2}, 1 \mu \mathrm{L}$ of $10 \times$ ExTaq buffer, $1 \mu \mathrm{L}$ of each primer, $1.5 \mu \mathrm{L}$ of template DNA (supernatant from the 1.5-mL centrifuge tube) and $14.8 \mu \mathrm{L}$ of $\mathrm{ddH}_{2} \mathrm{O}$ [34]. The amplification instrument was a PTC-200 DNA Engine (Hangzhou Bioer Technology Co. Ltd. Hangzhou, China). The following protocol was used for PCR amplification of the rDNA-ITS region: initial denaturation at $94{ }^{\circ} \mathrm{C}$ for $5 \mathrm{~min}, 40$ cycles of $94{ }^{\circ} \mathrm{C}$ for $50 \mathrm{~s}, 49^{\circ} \mathrm{C}$ for $40 \mathrm{~s}$ and $72{ }^{\circ} \mathrm{C}$ for $1 \mathrm{~min}$; and a final extension period at $72{ }^{\circ} \mathrm{C}$ for $7 \mathrm{~min}$ [35]. The PCR products of nematodes were further DNA sequenced by Jie Li Biomedical Technology Co., Ltd. (Shanghai, China).

\subsection{Experiments on Feeding Transmission by M. saltuarius}

One hundred and ten newly emerged adults were transferred into individual cages $(30 \times 40 \times 50 \mathrm{~cm})$ constructed of $0.2-\mathrm{cm}$ wire mesh, provided with fresh twigs, and kept at room temperature. Fifty beetles were provided P. koraiensis twigs as food and the other 60 beetles were provided non-Pinus conifer twigs as food ( 6 beetles per tree species). To prepare beetle food for the 
experiments, fresh twigs were clipped from P. koraiensis, L. principis, L. olgensis, T. chinensis, Picea pungens, Picea asperata, J. formosana, S. chinensis, A. fabri, A. holophylla and Podocarpus macrophyllus (including the new annual part and the two-year-old part) and cut into sections (15-cm in length). Each twig section was washed with running tap water and placed in the experimental cage as the food source for the beetle. Each twig was separately placed in three cages for repetition. New twigs were placed in the cages every 3 days until the beetles died. The 3-day feeding area (where the bark had been chewed) on each non-Pinus conifer twig was measured using transparent square millimeter paper [36] and the replaced twigs were extracted by the Baermann funnel technique. The amount of non-Pinus conifer bark consumed $\left(\mathrm{mm}^{2}\right)$ was used as the response variable for the analysis of feeding preference. The number of PWNs in a beetle was the sum of the number of PWNs transmitted into the twigs and the number of PWNs isolated after the beetle was dead.

To further verify the ability of the beetle to transmit PWNs, ten 5-year-old P. koraiensis potted seedlings (approximately $110 \mathrm{~cm}$ tall) without disease were selected on July 4 , and these trees were placed in two experimental cages $(250 \mathrm{~cm} \times 250 \mathrm{~cm} \times 270 \mathrm{~cm})$. One cage contained 15 beetles, and the other cage without beetles was used as a control. The seedlings were examined weekly for disease symptoms, which included wilting leaf discoloration and leaf fall. After the seedlings died, stem sections were cut from seedlings at each of the following positions: upper part (main shoot), middle part, lower part (above ground level), needles, branches and roots. Stem samples were sliced thin and placed on Baermann funnels to extract nematodes.

\subsection{Data Analysis}

One-way analysis of variance (ANOVA) was used to determine the significance of the mean differences in the number of nematodes separated from beetles. GraphPad Prism (8.0.1 for Windows) was used for chart construction.

\section{Results}

\subsection{Emergence of M. saltuarius and Identification of Nematodes}

The first M. saltuarius beetle emerged from the P. koraiensis timbers in the cage on May 9, and the full emergence period of the beetles lasted 49 days. During this period in 2018, the average temperature and humidity were $19{ }^{\circ} \mathrm{C}\left(11.0-25.0{ }^{\circ} \mathrm{C}\right)$ and $60.0 \%$ (30.0-90.0\%), respectively. We collected 279 adults, and their sex ratio was almost balanced (1.08:1; 145 males and 134 females). During the initial 5 days, only a few beetles emerged. However, nematode-carrying beetles accounted for a high proportion of the total number of beetles that emerged (Figure 1), and this proportion decreased over time.

Sequences were obtained from 10 selected nematodes, and the product length of the rDNA-ITS region was 916 bp according to the sequencing and Blast search in NCBI GenBank, which was $99-100 \%$ similar to that of B. xylophilus (https://www.ncbi.nlm.nih.gov/nucleotide/MN006173.1/).

The average number of nematodes per beetle was $7970 \pm 327$. Eight female beetles carried an average of 8010 nematodes, while 8 male beetles carried an average of 7920 nematodes, and the numbers carried by females and males did not significantly differ. Based on the number of extracted nematodes, the numbers of nematodes carried by male and female $M$. saltuarius beetles were similar with no significant difference. 


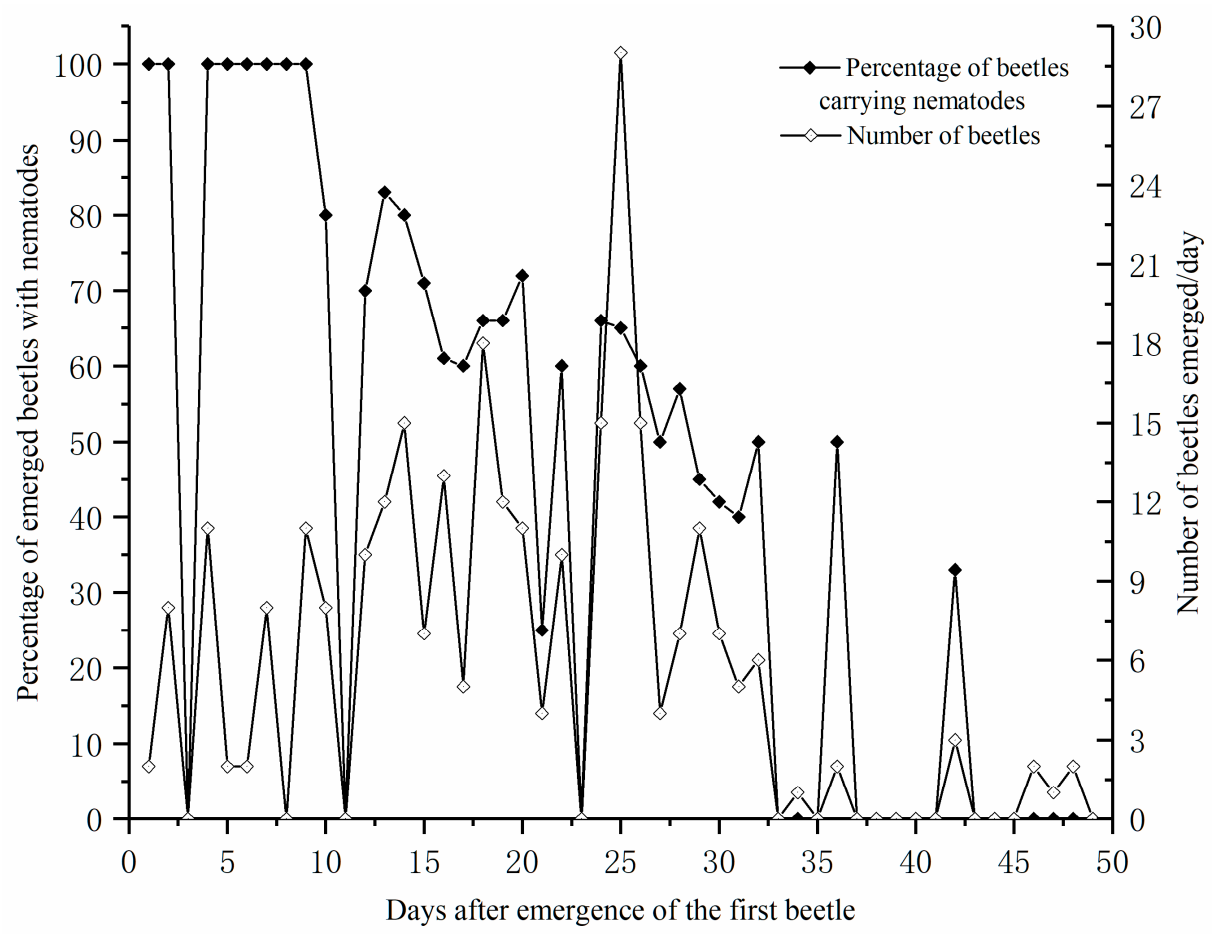

Figure 1. The percentage of beetles carrying nematodes $(\diamond)$ and the emergence of Monochamus saltuarius from pine timbers $(\diamond)$ during the emergence period in 2018.

\subsection{Types of Transmission of PWNs into Pine Twigs through M. saltuarius Feeding}

The transmission of PWNs to fresh pine twigs as indicated by the number of nematodes carried by the beetles was studied in further detail. Some beetles died prematurely during the test, so some valid data were selected. The details for the transmission of nematodes by individual beetles are shown in Table 1. The transmission of nematodes started from the 6th day after adult emergence. The first batch of nematodes was extracted from the feeding wounds after the beetles emerged and started their maturity feeding (e.g., beetle 7). This condition proved that $M$. saltuarius can transmit PWNs by feeding.

During the entire feeding period, the maximum number of nematodes transmitted by a beetle to the twigs was 550 (beetle 9), and the maximum number of nematodes extracted from a dead beetle was 21,000 (beetle 4). The number of nematodes transmitted to branches ranged from 1 to 550 within three days.

\subsection{Experiment of M. saltuarius Feeding on Potted Seedlings}

In this experiment involving feeding on potted seedlings, two of the tested trees in the experimental cage with M. saltuarius died on August 31, and PWNs were detected. The others died on October 3 and October 26, and PWNs were also found in these dead trees. Among the four dead trees, PWNs were found in all the trunks and branches, as well as in the needles of three experimental trees (Nos. 1,3 and 5). Three of the five experimental trees had PWNs in the roots (Nos. 1, 2 and 3). Conversely, none of the control trees died because these trees were placed in the experimental cage without beetles (Table 2).

\subsection{Transmission of PWNs by Mature M. saltuarius Feeding on Non-Pinus Conifer Tree Species}

In all 10 non-Pinus conifers, 60 beetles fed under compulsory feeding conditions and successfully transmitted PWNs. Among the 10 conifer species, the species preferred by M. saltuarius were Picea pungens, Picea asperata and A. fabri. The order of beetle feeding preference (as determined by comparing the size of the feeding area) was as follows: Picea asperata $>$ Picea pungens $>$ A. fabri 
$>$ A. holophylla $>$ L. principis $>$ J. formosana $>$ L. olgensis $>$ S. chinensis $>$ Podocarpus macrophyllus $>$ T. chinensis (Table 3).

Table 1. Summary of Bursaphelenchus xylophilus transmission by several Monochamus saltuarius individuals feeding on twigs of Pinus koraiensis during the entire feeding period.

\begin{tabular}{ccccccc}
\hline $\begin{array}{c}\text { Code } \\
\text { of } \\
\text { Beetles }\end{array}$ & $\begin{array}{c}\text { First Detection } \\
\text { of Nematodes } \\
\text { on Twig (Days } \\
\text { after Beetle } \\
\text { Emergence) }\end{array}$ & $\begin{array}{c}\text { Total } \\
\text { Number of } \\
\text { Nematodes } \\
\text { Transmitted } \\
\text { to Twig }\end{array}$ & $\begin{array}{c}\text { Number of } \\
\text { Nematodes } \\
\text { Retained in } \\
\text { Dead Beetle }\end{array}$ & $\begin{array}{c}\text { Number of } \\
\text { Nematodes } \\
\text { Transmitted } \\
\text { Per Day } \\
\text { (Max-Min) }\end{array}$ & $\begin{array}{c}\text { Last Detection } \\
\text { of Nematodes } \\
\text { (Days after } \\
\text { Beetle } \\
\text { Emergence) }\end{array}$ & $\begin{array}{c}\text { Number of Days of } \\
\text { Nematode } \\
\text { Presence in the } \\
\text { Feeding Wound } \\
\text { during the Entire } \\
\text { Feeding Period }\end{array}$ \\
\hline 1 & 12 & 49 & 0 & $21-1$ & 48 & 21 \\
2 & 18 & 64 & 0 & $50-2$ & 48 & 18 \\
3 & 9 & 100 & 5 & $43-1$ & 27 & 12 \\
4 & 9 & 118 & 21,000 & $116-2$ & 21 & 21 \\
5 & 15 & 63 & 33 & $38-1$ & 48 & 18 \\
6 & 12 & 44 & 586 & $23-1$ & 24 & 21 \\
7 & 6 & 69 & 0 & $22-1$ & 33 & 12 \\
8 & 9 & 69 & 3450 & $27-1$ & 36 & 27 \\
9 & 15 & 550 & 0 & $550-550$ & 9 & 24 \\
10 & 15 & 20 & 0 & $9-1$ & 21 & 2 \\
\hline
\end{tabular}

Table 2. Number of Bursaphelenchus xylophilus isolated from treated trees in the experimental cage. Data represent the means \pm SD of three replicates.

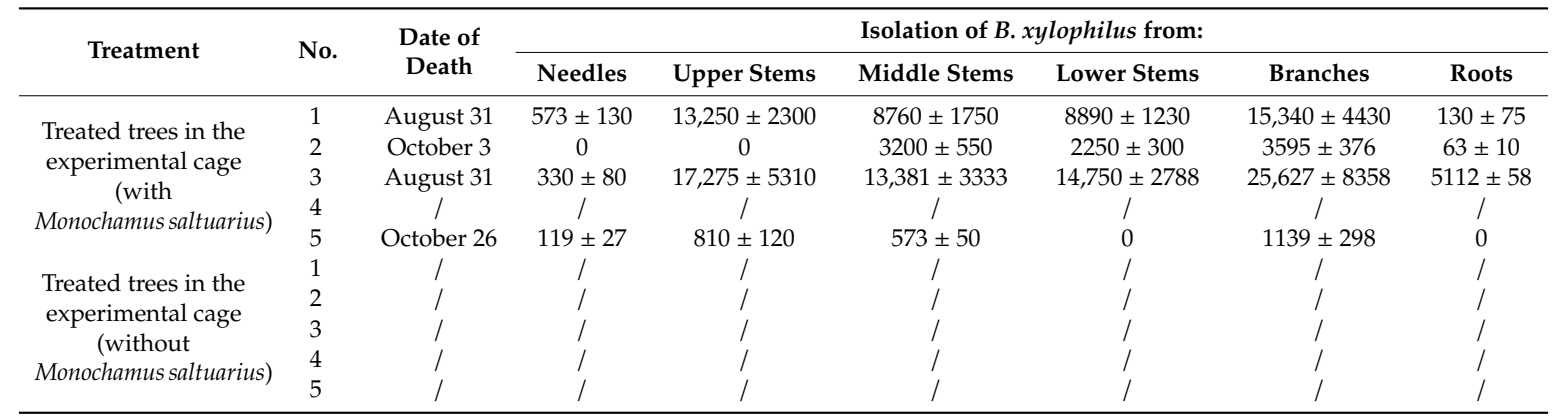

Table 3. Bark area consumed by Monochamus saltuarius and the number of Bursaphelenchus xylophilus entering twigs in 3 days. Data represent the means \pm SD of three replicates. Means in the column followed by the same letter did not differ significantly at $p<0.05$ (method of multiple comparisons).

\begin{tabular}{|c|c|c|}
\hline Tree Species & $\begin{array}{l}\text { Bark Area Consumed/ } \\
3 \text { Days }\left(\mathrm{mm}^{2}\right)\end{array}$ & $\begin{array}{c}\text { Number of Bursaphelenchus xylophilus } \\
\text { Entering Twigs in } 3 \text { Days }\end{array}$ \\
\hline Picea asperata * & $(694 \pm 7) \mathrm{a}$ & $(11 \pm 1) \mathrm{a}$ \\
\hline Picea pungens * & $(650 \pm 12) a$ & $(17 \pm 3) \mathrm{a}$ \\
\hline Abies fabri * & $(517 \pm 15) a$ & $(13 \pm 5) a$ \\
\hline Abies holophylla* & $(444 \pm 10) a$ & $(11 \pm 2) a$ \\
\hline Juniperus formosana & $(316 \pm 7) b$ & $(11 \pm 3) \mathrm{a}$ \\
\hline Sabina chinensis & $(308 \pm 5) b$ & $(16 \pm 1) \mathrm{a}$ \\
\hline Larix principis & $(335 \pm 20) b$ & $(11 \pm 9) \mathrm{a}$ \\
\hline Larix olgensis & $(314 \pm 18) b$ & $(17 \pm 1) \mathrm{a}$ \\
\hline Podocarpus macrophyllus & $(205 \pm 4) c$ & $(13 \pm 2) a$ \\
\hline Taxus chinensis $\mathbf{\Delta}$ & $(139 \pm 3) c$ & $(9 \pm 6) \mathrm{a}$ \\
\hline$F$ & 736.6 & 1.421 \\
\hline$P$ & $<0.0001$ & 0.244 \\
\hline
\end{tabular}

*: The favorite species of Monochamus saltuarius among the ten conifer species. ${ }^{\mathbf{4}}$ : The unpalatable species for Monochamus saltuarius among the ten conifer species. 


\section{Discussion}

In China, no research has been conducted on the transmission of PWNs by M. saltuarius because this species has not been reported to carry PWNs in mainland China. This vector of PWNs has received considerable attention since the outbreak of PWD in Fengcheng City in 2017. The above results showed that the young trees of $P$. koraiensis died due to PWD because they were fed on by M. saltuarius carrying PWNs in vivo. Under the climate conditions of Fengcheng City, PWNs can migrate from dead wood to $M$. saltuarius and break away from M. saltuarius during the feeding process to invade healthy pine trees. Therefore, our experiment showed for the first time that $M$. saltuarius beetles are carriers and disseminators of PWNs in China, thus providing a basis for the early detection and prevention of PWD in this area. On the sixth day after eclosion, the beetles began to transmit nematodes into the twigs. The transmission period of nematodes lasted up 48 days after beetle emergence. Tree No. 4 in the experimental cage with $M$. saltuarius showed little mechanical damage due to maturation feeding, which may explain why no PWNs specimens recovered (insufficient B. xylophilus invasion) [37]. The geographical environment and temperature differ substantially from southern to northern China. Cold stress may influence the expansion of PWD and the reproduction of M. alternatus in China [38]. Previous studies have shown that the areas suitable for the development of PWD are mainly in the southeast of China and the northernmost areas in the southern part of Beijing, Tianjin and southern Hebei [21,39]. However, PWD has been found in Liaoning Province, where M. saltuarius is distributed, but no $M$. alternatus have been found. According to previous studies, M. alternatus is a native sawyer beetle in China, although PWD did not occur and spread in China until 1982 [40], indicating that although $M$. alternatus can transmit PWN as a vector, there were no PWNs in mainland China at that time. Therefore, PWD had not occurred. M. saltuarius is also a native longhorn beetle in northern China. There are many possible reasons for the occurrence of PWNs in Fengcheng, Liaoning Province. The most likely scenario is that pine wood products infected with PWN in southern China were artificially transported to Fengcheng City, and M. alternatus then emerged from these trees and fed on the local Korean pine. However, M. alternatus could not survive the low winter temperatures (the annual average temperature is below $10^{\circ} \mathrm{C}$ ) and thus died [41]. However, PWNs can tolerate low temperatures [42] and survive until M. saltuarius eclosion in the following year, thus, expanding their range and dispersing. The alien PWN adopted the native $M$. saltuarius as a novel vector in this newly invaded area [43].

Northeast China is rich in tree species and conifers. Our experiments showed that $M$. saltuarius not only feeds on Korean pine but can also spread PWNs to other conifer trees. Moreover, Taxus, Juniperus, Sabina and Podocarpus trees are new species that can be fed on by M. saltuarius. Picea pungens, Picea asperata, A. holophylla, L. principis-rupprechtii, and L. olgensis are new species of conifer trees that can be infected with PWD. Interestingly, after approximately $20 \mathrm{~min}$ of feeding on T. chinensis, M. saltuarius slowed and even entered a state of pseudo death (shock), which may have been caused by the chemical composition of $T$. chinensis. The beetles then recovered after approximately half a day. After recovery, $M$. saltuarius continued feeding and then repeated the process. The PWNs successfully developed into adults in T. chinensis and were not affected. We urgently need to increase awareness regarding the prevention of PWD. Pine massoniana was originally considered the disease-resistant tree species when PWD first occurred in Nanjing, but the main pine species damaged by PWN in southeast China has recently changed from $P$. thumbergii to $P$. massoniana [44].

\section{Conclusions}

The present study showed that M. saltuarius was a vector of PWD in northeast China. On the sixth day after eclosion, the beetle began to transmit nematodes into the twigs. The transmission period of nematodes lasted up 48 days after beetle emergence. M. saltuarius not only feeds on pines, but also spreads PWN to other conifers. The tree species preferred by M. saltuarius for feeding are P. koraiensis, Picea pungens, Picea asperata, and Abies fabri. Currently, PWNs have spread to Liaoning Province, reflecting the inevitability that PWNs will be detected in Jilin Province, Heilongjiang Province and 
other high-latitude regions in China. It is important to raise awareness in order to prevent the disease from expanding and to provide a reference for the control of PWD.

Author Contributions: Conceptualization, M.L.; Data curation, M.L.; Formal analysis, S.-H.S.; Funding acquisition, F.-M.C.; Methodology, H.L., H.S. and F.-M.C.; Project administration, F.-M.C.; Software, M.L.; Validation, R.-C.S.; Writing-original draft, M.L.; Writing—review and editing, M.L. All authors contributed to the final draft. All authors have read and agreed to the published version of the manuscript.

Funding: This research was funded by the National Key R \& D Program of China (Grant No. 2017YFD0600104) and the Shenyang Science and Technology Planning Project (Grant No. 18-400-3-03).

Conflicts of Interest: The authors declare no conflict of interest.

\section{References}

1. Mamiya, Y. Pathology of the Pine Wilt Disease Caused by Bursaphelenchus xylophilus. Annu. Rev. Phytopathol. 1983, 21, 201-220. [CrossRef] [PubMed]

2. Mamiya, Y. Pine wilting disease caused by the pine wood nematode, Bursaphelenchus lignicolus, in Japan. JARQ (Jpn. Agric. Res. Quart) 1976, 10, 206-211.

3. Yang, B.J.; Wang, Q.L. Distribution of the pinewood nematode in China and susceptibility of some Chinese and exotic pines to the nematode. Can. J. For. Res. 1989, 19, 1527-1530.

4. La, Y.J.; Moon, Y.S.; Yeo, W.H.; Shin, S.C.; Bak, W.C. Recent status of pine wilt disease in Korea. In Proceedings of the Sustainability of Pine Forests in Relation to Pine Wilt \& Decline International Symposium, Tokyo, Japan, 27-28 October 1998.

5. Sun, Y.C. First report of pine wilt disease from Pinus thunbergii Parl in Sun Yat-sen Mausoleum. J. Jiangsu For. Sci. Technol. 1982, 2, 27.

6. Zhao, B.G. Pine wilt disease in China. In Pine Wilt Disease; Springer: Berlin/Heidelberg, Germany, 2008; pp. 18-25.

7. Linit, M.J.; Kondo, E.; Smith, M.T. Insects associated with the pinewood nematode, Bursaphelenchus xylophilus (Nematoda: Aphelenchoididae), in Missouri. Environ. Entomol. 1983, 12, 467-470. [CrossRef]

8. Linit, M.J. Nemtaode-vector relationships in the pine wilt disease system. J. Nematol. 1988, $20,227$.

9. Linit, M.J. Transmission of Pinewood Nematode Through Feeding Wounds of Monochamus carolinensis (Coleoptera: Cerambycidae). J. Nematol. 1990, 22, 231-236.

10. Bergdahl, D.; Halik, S.; Tomminen, J.; Akar, H. Frequency of infestation of Monochamus notatus and M. scutellatus by Bursaphelenchus xylophilus in Vermont. Phytopathology 1991, 81, 120.

11. Luzzi, M.; Wilkinson, R.; Tarjan, A. Transmission of the pinewood nematode, Bursaphelenchus xylophilus, to slash pine trees and $\log$ bolts by a cerambycid beetle, Monochamus titillator, in Florida. J. Nematol. 1984, $16,37$.

12. Akbulut, S.; Stamps, W. Insect vectors of the pinewood nematode: A review of the biology and ecology of Monochamus species. For. Pathol. 2012, 42, 89-99. [CrossRef]

13. Wingfield, M.; Blanchette, R.A. The pine-wood nematode, Bursaphelenchus xylophilus, in Minnesota and Wisconsin: Insect associates and transmission studies. Can. J. For. Res. 1983, 13, 1068-1076. [CrossRef]

14. Mamiya, Y.; Enda, N. Transmission of bursaphelenchus lignicolus (nematoda: Aphelenchoididae) by monochamus alternatus (coleoptera: Cerambycidae). Nematologica 1972, 18, 159-162. [CrossRef]

15. Sato, H.; Takeshi, S.; Kobayashi, M. Transmission of Bursaphelenchus xylophilus (Steiner et Buhrer) Nickle (Nematoda, Aphelenchoididae) by Monochamus saltuarius (Gebler)(Coleoptera, Cerambycidae). J. Jpn. For. Soc. 1987, 69, 492-496.

16. Haran, J.; Rousselet, J.; Tellez, D.; Roques, A.; Roux, G. Phylogeography of Monochamus galloprovincialis, the European vector of the pinewood nematode. J. Pest Sci. 2018, 91, 247-257. [CrossRef]

17. Pajares, J.; Álvarez, G.; Hall, D.; Ibarra, N.; Hoch, G.; Halbig, P.; Cocoş, D.; Johansson, H.; Schroeder, M. Attractants for management of the pine sawyer beetle Monochamus sutor, a potential vector of Bursaphelenchus xylophilus. J. Appl. Entomol. 2017, 141, 97-111. [CrossRef]

18. Togashi, K.; Taga, Y.; Iguchi, K.; Aikawa, T. Bursaphelenchus mucronatus (Nematoda: Aphelenchoididae) vectored by Monochamus urussovi (Coleoptera: Cerambycidae) in Hokkaido, Japan. J. For. Res. 2008, 13, 127-131. [CrossRef] 
19. Akbulut, S.; Keten, A.; Stamps, W.T. Population dynamics of Monochamus galloprovincialis Olivier (Coleoptera: Cerambycidae) in two pine species under laboratory conditions. J. Pest Sci. 2008, 81, 115-121. [CrossRef]

20. Pajares, J.A.; Álvarez, G.; Ibeas, F.; Gallego, D.; Hall, D.R.; Farman, D.I. Identification and field activity of a male-produced aggregation pheromone in the pine sawyer beetle, Monochamus galloprovincialis. J. Chem. Ecol. 2010, 36, 570-583. [CrossRef]

21. Li, Y.X. Analysis on the trend of invasion and expansion of Bursaphelenchus xylophilus. For. Pest Dis. 2018, 1, 1-4.

22. Naves, A.C.G.P. Morphobiometric and Molecular Characterization of Bursaphelenchus Fuchs, 1937 (Nematoda: Aphelenchoididae) Species Associated with Pinus Pinaster Ainton in Portugal. Ph.D. Thesis, Universidade de Évora Portugal, Évora, Portugal, 2008.

23. Koo, C.D.; Lee, H.Y.; Han, J.H.; Sung, J.H.; Shin, J.H. Infection behavior and distribution of Bursaphelenchus xylophilus in Pinus densiflora trees. For. Sci. Technol. 2013, 9, 81-86.

24. Han, J.W.; Kim, H.K.; Kang, W.J.; Kim, G.A. Feeding and oviposition preference of the Sakhalin pine sawyer Monochamus saltuarius (Coleoptera: Cerambycidae) for various tree species. Entomol. Res. 2016, 46, 331-336. [CrossRef]

25. Yu, W.Y.; Zhang, E.Y.; Zhi, Q. Monochamus saltuarius harms Pinus banksiana. Plant Prot. 1992, 18, 51.

26. Han, H.; Chung, Y.J.; Shin, S.C. First report of pine wilt disease on Pinus koraiensis in Korea. Plant Dis. 2008, 92, 1251. [CrossRef] [PubMed]

27. Nunes da Silva, M.; Lima, M.R.M.; Vasconcelos, M.W. Susceptibility evaluation of Picea abies and Cupressus lusitanica to the pine wood nematode (Bursaphelenchus xylophilus). Plant Pathol. 2013, 62, 1398-1406. [CrossRef]

28. Shi, J.; Chen, F.; Luo, Y.Q.; Wang, Z.; Xie, B.Y. First isolation of pine wood nematode from Pinus tabuliformis forests in China. For. Pathol. 2013, 43, 59-66. [CrossRef]

29. Nakamura, K.; Akiba, M.; Kanetani, S. Pine wilt disease as promising causal agent of the mass mortality of Pinus armandii Franch. var. amamiana (Koidz.) Hatusima in the field. Ecol. Res. 2001, 16, 795-801. [CrossRef]

30. Zhang, X.; Stamps, W.T.; Linit, M.J. A nondestructive method of determining Bursaphelenchus xylophilus infestation of Monochamus spp. vectors. J. Nematol. 1995, 27, 36.

31. Shuto, Y.; Watanabe, H. Attractants from a vector, Monochamus alternatus, for the pine wood nematode. Agric. Biol. Chem. 1987, 51, 1457-1458.

32. Vrain, T.C.; Wakarchuk, D.A.; Levesque, A.C.; Hamilton, R.I. Intraspecific rDNA restriction fragment length polymorphism in the Xiphinema americanum group. Fundam. Appl. Nematol. 1992, 15, 563-573.

33. Ferris, V.R. Variation in spacer ribosomal DNA in some cyst-forming species of plant parasitic nematodes. Fundam. Appl. Nematol. 1993, 16, 177-184.

34. Chen, F.M.; Ye, J.R.; Tang, J.; Wu, X.Q. A Study on Detection Technique of Bursaphelenchus xylophilus and B. mucronatus by RAPD. J. Nanjing For. Univ. 2005, 29, 25-28.

35. Green, M.; Rott, M.; Leal, I.; Humble, L.; Allen, E. Application of a real-time PCR method for the detection of pine wood nematode, Bursaphelenchus xylophilus, in wood samples from lodgepole pine. Nematology 2007, 9, 351-362. [CrossRef]

36. Li, R.J.; Xu, F.Y.; Zhang, P. Host Preference by Monochamus alternatus (Hope) during Maturation Feeding on Pine Species and Masson Pine Provenances. Chin. For. Sci. Technol. 2003, 2, 91-98.

37. Kubozono, M.; Sone, K.; Kawauchi, H.; Tsuji, M. Resistance to the pine wilt disease and initial invasion of the pine wood nematode, Bursaphelenchus xylophilus (Steiner et Buhrer) Nickle, into seedlings of resistant clones of Pinus thunbergii Parl. Bull. Kagoshima Univ. For. 1998, 26, 37-41.

38. Ohsawa, M.; Akiba, M. Possible altitude and temperature limits on pine wilt disease: The reproduction of vector sawyer beetles (Monochamus alternatus), survival of causal nematode (Bursaphelenchus xylophilus), and occurrence of damage caused by the disease. Eur. J. For. Res. 2014, 133, 225-233.

39. Zhang, K. Research Advances of Pine Wood Nematode Disease in China. World For. Res. 2010, $23,59-63$.

40. Huang, J.; Zhang, J.; Li, M.J.; Xia, T.F. Seasonal variations in the incidence of Monochamus alternatus adults (Coleoptera: Cerambycidae) and other major Coleoptera: A two-year monitor in the pine forests of Hangzhou, Eastern China. Scand. J. For. Res. 2015, 30, 507-515. [CrossRef]

41. Du, H.B.; Wu, Z.F.; Zhang, N.; Zong, S.W.; Meng, X.J. Characteristics of Extreme Temperature and Precipitation Events over Dandong During the Last Six Decades. Sci. Geogr. Sin. 2013, 33, 473-480. 
42. Zhao, L.L.; Wei, W.; Zhang, X.Y.; Kulhavy, D.; Sun, J.H. Low temperature induces two growth-arrested stages and change of secondary metabolites in Bursaphelenchus xylophilus. Nematology 2007, 9, 663-670. [CrossRef]

43. Sheng, M.L. Two new species of the genus Ischnoceros Gravenhorst (Hymenoptera, Ichneumonidae) parasitizing woodborers with a key to species of Ischnoceros known in China. Acta Zootaxonomica Sin. 2008, 33, 508-513.

44. Zhen, D.; Xu, F.Y.; Ge, M.H.; Zhu, K.G. Studies on the Relationship of Amino Acid Content of Different Masson Pine Provenances and Their Resistance to Pine Wood Nematode (PWN). For. Res. 1998, 3, 6-12.

(C) 2020 by the authors. Licensee MDPI, Basel, Switzerland. This article is an open access article distributed under the terms and conditions of the Creative Commons Attribution (CC BY) license (http://creativecommons.org/licenses/by/4.0/). 